\title{
Stratospheric influence on baroclinic lifecycles and its connection to the Arctic Oscillation
}

\author{
Matthew A. H. Wittman \\ Department of Applied Physics and Applied Mathematics, Columbia University, New York, New York, USA \\ Lorenzo M. Polvani \\ Department of Applied Physics and Applied Mathematics and Department of Earth and Environmental Sciences, Columbia \\ University, New York, New York, USA
}

Richard K. Scott

NorthWest Research Associates, Inc., Bellevue, Washington, USA

Andrew J. Charlton

Department of Applied Physics and Applied Mathematics, Columbia University, New York, New York, USA

Received 12 May 2004; revised 28 June 2004; accepted 29 July 2004; published 25 August 2004.

[1] Using an idealized primitive equation model, we investigate how stratospheric conditions alter the development of baroclinic instability in the troposphere. Starting from the lifecycle paradigm of Thorncroft et al., we consider the evolution of baroclinic lifecycles resulting from the addition of a stratospheric jet to the LC1 initial condition. We find that the addition of the stratospheric jet yields a net surface geopotential height anomaly that strongly resembles the Arctic Oscillation. With the additional modification of the tropospheric winds to resemble the high-AO climatology, the surface response is amplified by a factor 10 and, though dominated by the tropospheric changes, shows similar sensitivity to the stratospheric conditions. INDEX TERMS: 3334 Meteorology and Atmospheric Dynamics: Middle atmosphere dynamics (0341, 0342); 3337 Meteorology and Atmospheric Dynamics: Numerical modeling and data assimilation; 3362 Meteorology and Atmospheric Dynamics: Stratosphere/troposphere interactions. Citation: Wittman, M. A. H., L. M. Polvani, R. K. Scott, and A. J. Charlton (2004), Stratospheric influence on baroclinic lifecycles and its connection to the Arctic Oscillation, Geophys. Res. Lett., 31, L16113, doi:10.1029/2004GL020503.

\section{Introduction}

[2] Since the discovery of the downward propagation of the Arctic Oscillation (AO) [Baldwin and Dunkerton, 1999], there has been intense speculation as to which dynamical mechanisms explain how the troposphere responds to the stratospheric state. A number of mechanisms have been proposed, including direct adjustment via PV-inversion [Hartley et al., 1998], planetary wave reflection [Perlwitz and Harnik, 2003], and QBO-like wave-mean flow interaction [Plumb and Semeniuk, 2003]. The common point of these is that they all focus on the planetary-scale response to the stratospheric state.

[3] However, it is quite clear that while the structure of the $\mathrm{AO}$ in the stratosphere corresponds well to variations in

Copyright 2004 by the American Geophysical Union. 0094-8276/04/2004GL020503 the strength of the polar vortex, the structure of the AO in the troposphere represents an average over individual synoptic systems [Cash et al., 2002]. Furthermore, recent work [Polvani and Kushner, 2002] has shown that a surface AO-like response can be produced by perturbing the stratosphere in the absence of planetary waves, and a recent GCM study [Charlton et al., 2004] has produced synoptic scale tropospheric responses to changes in the strength of the stratospheric jet. Further hints at a mechanism are offered by Baldwin et al. [2003], that shows instantaneous shortwave momentum fluxes correlated with earlier values of the stratospheric AO index, and Shepherd [2002], that suggests the possibility of a sharp bifurcation in the location of upper atmosphere wave drag depending on the stratospheric state. These studies strongly suggest that stratospheric influence on the troposphere might be mediated by changes to the development of baroclinic eddies.

[4] To date, however, the only explicit study of this influence [Tanaka and Tokinaga, 2002] has focused on the differences in the linear growth rates of unstable modes under high- and low-AO conditions. Baroclinic instability is, however, a fundamentally non-linear phenomenon, consisting of lifecycles of eddy growth, saturation and decay. Furthermore, no attempt has been made to explicitly examine the relative effects of stratospheric and tropospheric anomalies on baroclinic development.

[5] To address the question of stratospheric influence we perform numerical experiments with single baroclinic lifecycles, following Thorncroft et al. [1993], hereafter THM. We solve initial value problems with a baroclinically unstable tropospheric jet, to which we add a stratospheric jet. By modifying the initial conditions in this way, we seek to answer the simple question: how do baroclinic lifecycles change in response to stratospheric zonal wind anomalies?

\section{Method}

[6] We use the BOB pseudo-spectral dynamical core [Rivier et al., 2002], which integrates the dry adiabatic global primitive equations. Results presented here are obtained at T170 spectral resolution, with 40 vertical levels 

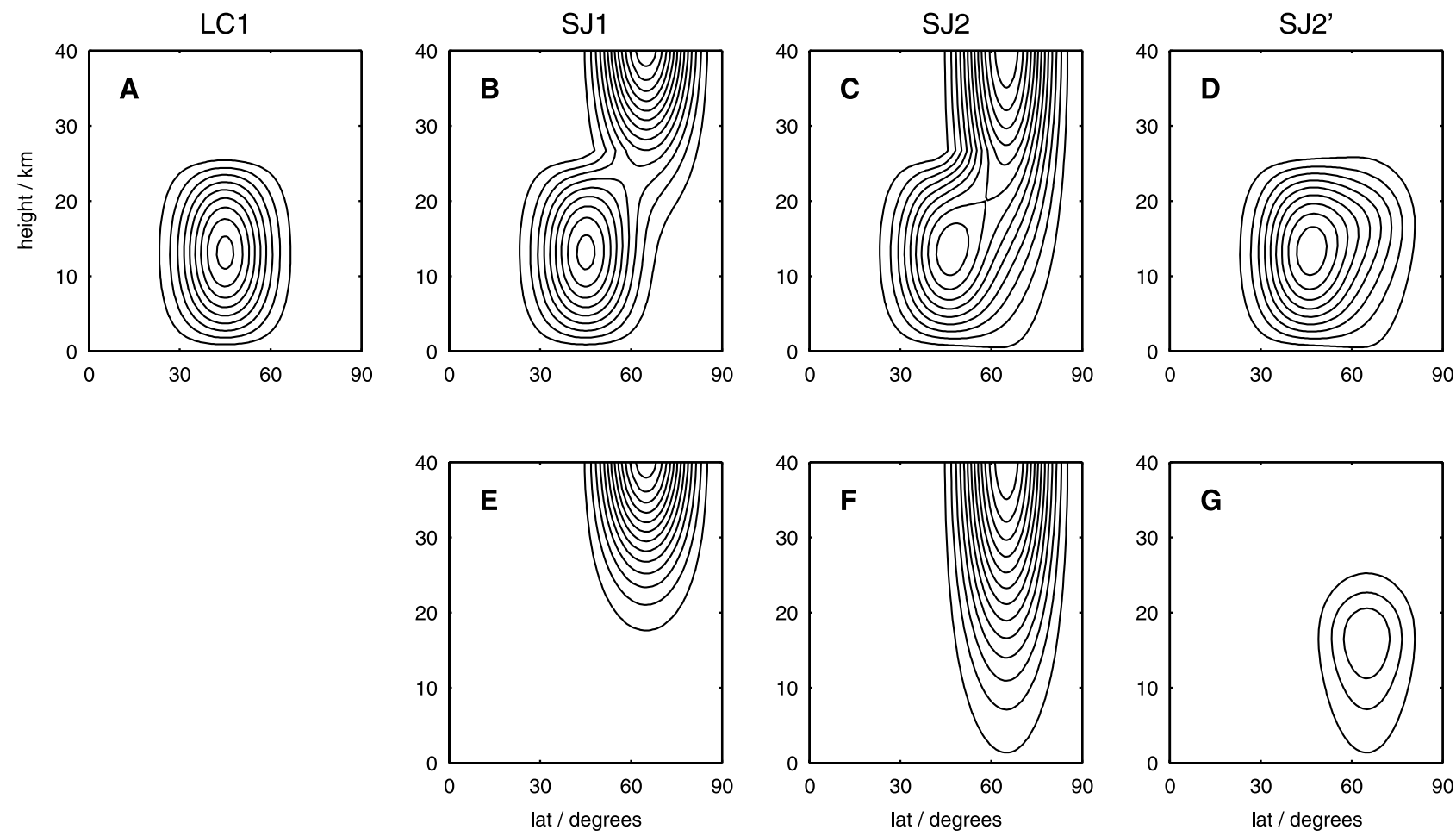

Figure 1. Initial zonal wind (top row) and differences with LC1 (bottom row) for the four cases discussed in this study. The contour interval is $5 \mathrm{~m} \mathrm{~s}^{-1}$, and the lowest contour is $5 \mathrm{~m} \mathrm{~s}^{-1}$.

arranged as in Polvani and Kushner [2002]; we have verified that the results discussed below are qualitatively independent of resolution. Initial conditions are specified as a zonal wind field in thermal wind balance with a temperature field whose meridional mean is the U.S. Standard Atmosphere [1976]. The initial zonal wind is constructed analytically as the sum of two jets: a tropospheric jet $u_{T}$ that closely matches that of THM (cf. their Figure 3a), and a stratospheric jet $u_{S}$, representing the polar vortex. An initial zonal wave number 6 perturbation of $1 \mathrm{~K}$ is applied to the temperature field, resulting in subsequent flow with wave-6 symmetry.

[7] Functional forms of $u_{T}$ and $u_{S}$ are given in Appendix A; jet locations and shapes are fully controllable by varying appropriate parameters. We have performed many integrations over the parameter space of these jets, and we present here the results of a small number of these, chosen as illustrative examples to highlight key results which are qualitatively robust.

\section{Results}

[8] In Figure 1a, we show the initial zonal winds for the reference case, labeled LC1, which is based on THM's LC1 initial conditions, and results in a lifecycle that closely matches that of THM. A mature stage of this lifecycle is presented in Figure 2a, where we plot the surface temperature at day 10 , after the instability has saturated and nonlinear wavebreaking has produced cyclones, visible as closed temperature contours.

[9] The influence of the stratosphere is illustrated by case SJ1, whose initial condition is shown in Figure $1 \mathrm{~b}$. In this case we add a jet confined to the stratosphere,
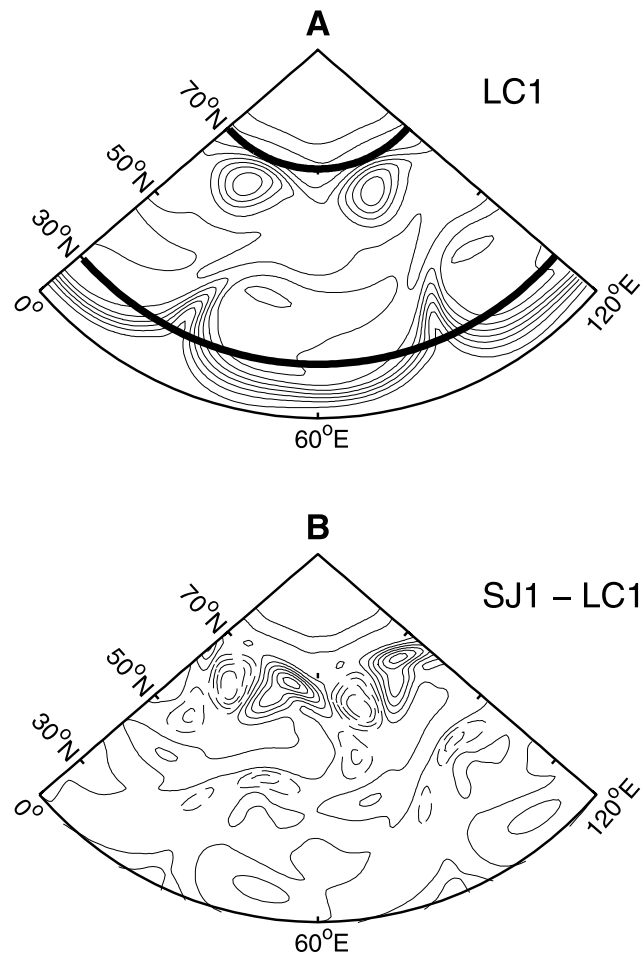

Figure 2. A: The surface temperature at day 10 for LC1. The contour interval is $10 \mathrm{~K}$. Solid lines mark the $70 \mathrm{~N}$ and $30 \mathrm{~N}$ meridians. B: The difference in surface temperature at day 10 between SJ1 and LC1. The contour interval is $2 \mathrm{~K}$, and negative values are dashed. 


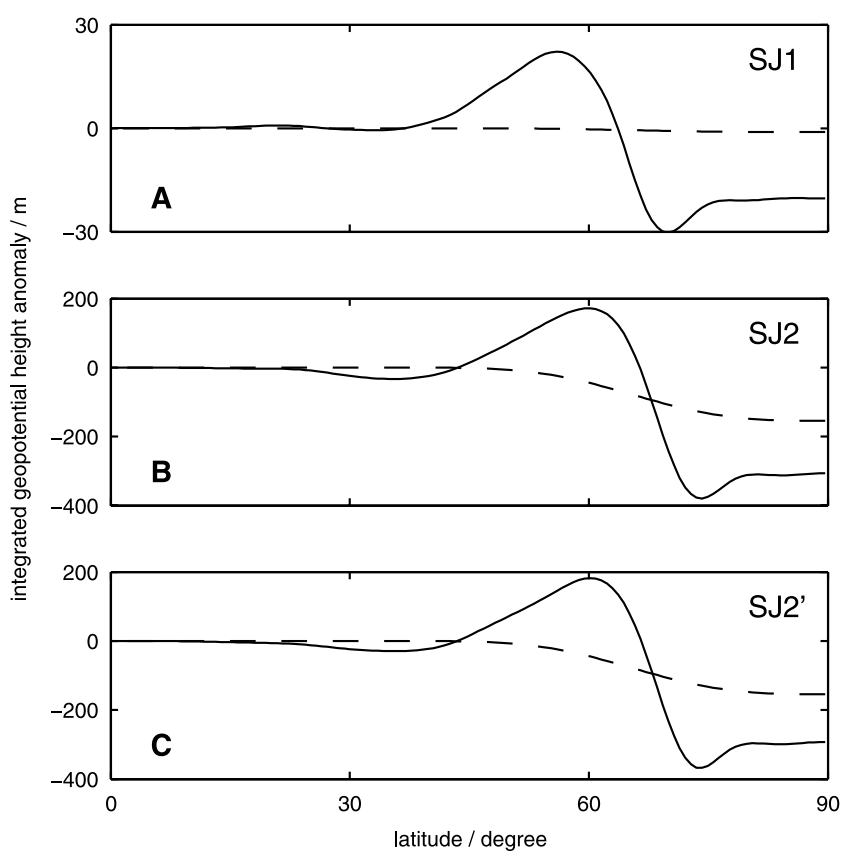

Figure 3. The zonally averaged surface geopotential height anomaly for cases SJ1 (A), SJ2 (B) and SJ2' (C). The solid lines are time averaged over 20 days of integration, and the dotted lines show the initial values.

corresponding to a high stratospheric AO index. Below $15 \mathrm{~km}$, the initial winds in SJ1 are nearly identical to those in LC1, as can be seen from the difference plot (Figure 1e). The day-10 surface temperature for case $\mathrm{SJ} 1$ is very similar to that of $\mathrm{LC} 1$; the departure from $\mathrm{LC} 1$ evolution is only visible in the difference map (Figure 2b), which consists of small dipoles at the same scale as the synoptic eddies, with a meridional gradient of $\sim 2 \mathrm{~K} /$ degree. Notice the (small) overall poleward displacement of synoptic systems due to the presence of the stratospheric jet. In Figure 3a, we show the zonal mean surface geopotential height anomaly, i.e., the difference from the LC1 value. While the initial anomaly (dashed line) is very small, the integral of the anomaly over 20 days (solid line), which gives a measure of the total response of the troposphere to the stratospheric jet, shows a dipole approximately of $\pm 25 \mathrm{~m}$, which clearly resembles the surface AO.

[10] The surface response to the stratosphere in case SJ1 is small: the $50 \mathrm{~m}$ peak-to-peak geopotential height dipole corresponds to a surface pressure dipole of $\sim 7 \mathrm{hPa}$. We next ask if the surface AO response is larger for a combined stratospheric and tropospheric initial anomaly, a feature seen in observations during the high-AO state [see, e.g., Tanaka and Tokinaga, 2002, Figure 11; Ambaum et al., 2001, Figure 6]. Case SJ2, shown in Figure 1c, has initial tropospheric winds that are modified to resemble the mean state corresponding to a high tropospheric AO index, in addition to the high stratospheric AO index retained from SJ1. As illustrated by the difference from LC1 (Figure 1f), the initial winds are then enhanced on the poleward side of the tropospheric jet. While one may anticipate that most of the tropospheric response to this modification to the initial conditions will be due to the presence of anticyclonic meridional shear across the baroclinic THM jet [Dong and
James, 1997a, 1997b], here we are interested in the distinct effect of the stratosphere.

[11] In case SJ2, the evolution of the lifecycle is markedly different from LC1, as shown by the day-10 surface temperature (Figure 4a). The cyclonic centers are shifted poleward by $10^{\circ}$, and a corresponding poleward shift in the low-latitude frontal features. The differences from LC1 (Figure $4 b$ ) are again at the synoptic scale, but have an amplitude approximately 2 times larger than the SJ1 differences. The corresponding integrated surface geopotential height anomaly (Figure 3b) shows a dipole similar to SJ1 but with a 10 -fold increase in magnitude.

[12] At this point one may ask how much of the observed difference to the surface response between $\mathrm{SJ} 2$ and LC1 is due to the tropospheric modifications to the LC1 initial condition, and how much to modifications in the stratosphere. To answer this question, we show a final lifecycle, labeled SJ2', with initial conditions shown in Figure 1d. In case $\mathrm{SJ} 2^{\prime}$ the initial winds match those of $\mathrm{SJ} 2$ in the troposphere and those of LC1 in the stratosphere. The difference in the resulting day 10 evolution from LC1 (Figure 5a) is nearly identical to the SJ2 - LC1 difference, and the resulting integrated surface geopotential anomaly (Figure 3c) is also very similar to that of SJ2. However, the difference between the SJ2 and SJ2' day 10 surface temperature fields (Figure 5b) reveals features at the synoptic scale, as in the SJ1 - LC1 difference (Figure 2b). Therefore even though the surface response in $\mathrm{SJ} 2$ and $\mathrm{SJ}_{2}^{\prime}$ is dominated by sensitivity to tropospheric anomalies, the sensitivity to stratospheric anomalies persists independently
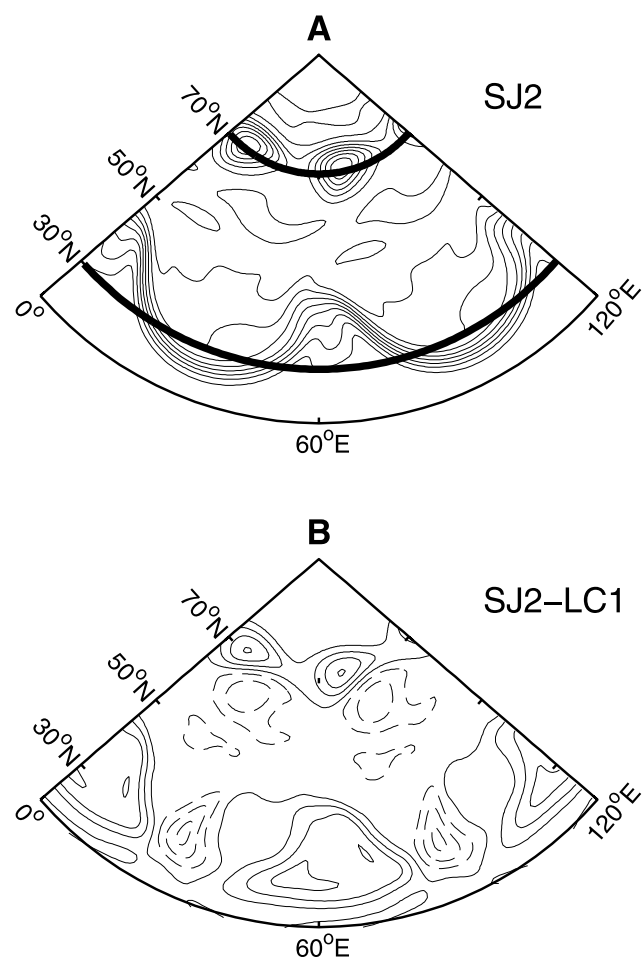

Figure 4. A: The surface temperature at day 10 for SJ2. The contour interval is $10 \mathrm{~K}$. Solid lines mark the $70 \mathrm{~N}$ and $30 \mathrm{~N}$ meridians. B: The difference in surface temperature at day 10 between SJ2 and LC1. The contour interval is $5 \mathrm{~K}$, and negative values are dashed. 

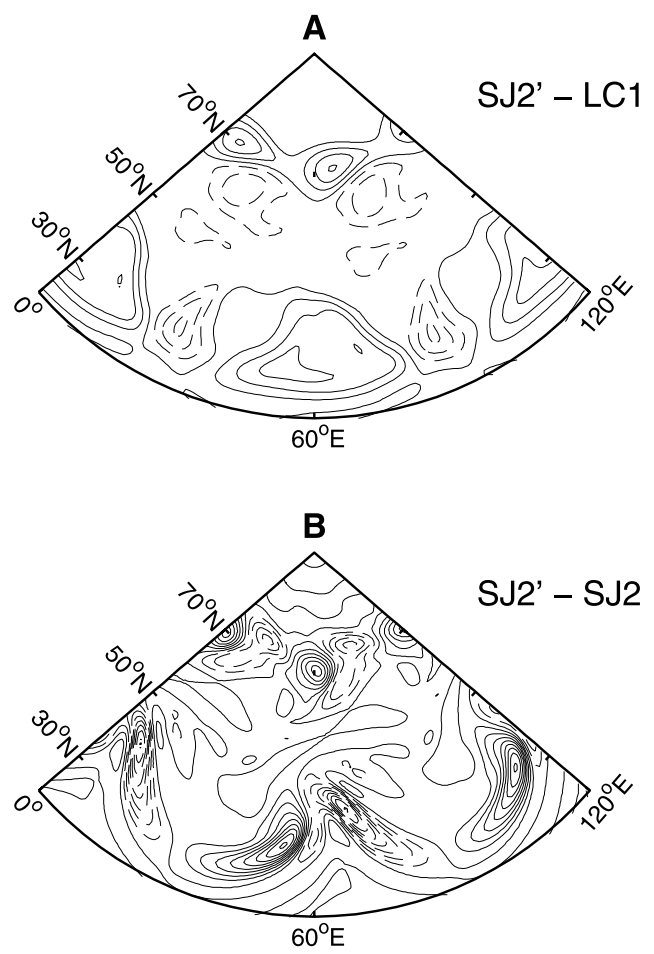

Figure 5. A: The difference in day-10 surface temperature between $\mathrm{SJ}^{\prime}{ }^{\prime}$ and $\mathrm{LC} 1$. The contour interval is $5 \mathrm{~K}$. B: The difference in day-10 surface temperature between SJ2 ${ }^{\prime}$ and SJ2. The contour interval is $1 \mathrm{~K}$. Negative values are dashed in both panels.

of the tropospheric initial state, and manifests itself at the synoptic scale.

\section{Discussion}

[13] Our numerical experiments show that baroclinic lifecycles are sensitive to the zonal mean stratospheric state across a range of tropospheric initial states.

[14] The occurrence of an AO-like surface geopotential height anomaly resulting from a stratospherically influenced baroclinic lifecycle, and the fact that the anomalies in the surface temperature field are at the synoptic scale, show how the stratosphere is able to influence large-scale tropospheric variability through a consistent effect on individual synoptic systems. Though the response of an individual lifecycle to stratospheric changes in the initial condition is weak compared with the response to tropospheric changes, the former may be significant when applied consistently over many lifecycles.

[15] The lifecycle response is greatly amplified when the jet added to the LC1 initial condition extends throughout the troposphere, as in $\mathrm{SJ} 2$, which is representative of an observed high-AO state. This amplification is due to the fact that the steering level of developing baroclinic eddies is below $5 \mathrm{~km}$, making them most sensitive to added meridional shears there [Hartmann, 2000; Dong and James, 1997a, 1997b].

[16] The enhanced nonlinear response in SJ2 and SJ2' corroborates the linear result of Tanaka and Tokinaga [2002], who suggest that baroclinic eddies might feed back into the high-AO mean flow. As can be inferred from
Figures $3 \mathrm{~b}$ and $3 \mathrm{c}$, the projection of the final integrated surface geopotential height anomaly onto a notional AO pattern is greater than that of the initial anomaly. This nonlinear eddy feedback has been noted before in the forceddissipative experiments of Kushner and Polvani [2004] and Song and Robinson [2004].

[17] In sum, we find that although tropospheric changes to the initial conditions yield much larger responses than stratospheric changes, a comparison of SJ1 with LC1, and $\mathrm{SJ}{ }^{\prime}$ with SJ2, reveals synoptic-scale differences due to the presence or absence of a jet in the stratosphere. These changes, although small, may be significant in the effort to explain the stratospheric influence on the troposphere demonstrated in recent papers.

\section{Appendix A: Initial Conditions}

[18] In coordinates $(\phi, z)$, latitude and log-pressure pseudo height with scale height $7 \mathrm{~km}$, the tropospheric jet is specified by

$$
u_{T}=u_{0 T} \sin \left(\frac{\pi z}{z_{\text {top }}}\right) \sin ^{3}\left\{\pi \sin ^{2}(\phi)\right\}
$$

and $u_{0 T}=46.6 \mathrm{~m} \mathrm{~s}^{-1}$ and $z_{\text {top }}=26.2 \mathrm{~km}$.

[19] The stratospheric jet is specified by

$$
u_{S}=\left\{\begin{array}{lc}
u_{0 S} \exp \left\{-\left(\frac{z-z_{0 S}}{z_{h w}}\right)^{2}\right\} & \text { for } \| \phi\left|-\phi_{0 S}\right| \\
\times \sin \left\{\pi \sin ^{2}\left[\frac{\pi}{4}\left(\frac{|\phi|-\phi_{0 S}}{\phi_{h w}}+1\right)\right]\right\} & \leq \phi_{h w} \\
0 & \text { otherwise }
\end{array}\right.
$$

with $u_{0 S}=70 \mathrm{~m} \mathrm{~s}^{-1}, \phi_{h w}=25^{\circ}, z_{0 S}=42 \mathrm{~km}$ and $\phi_{0 S}=65^{\circ}$; $z_{h w}=15 \mathrm{~km}$ for SJ1 and $z_{h w}=25 \mathrm{~km}$ for SJ2.

[20] The functional form of SJ2' is identical to that of SJ2 with $u_{S}$ multiplied by the vertical envelope function:

$$
f(z)=\frac{1}{2}\left\{1-\tanh \left(\frac{z-20}{5}\right)\right\}
$$

[21] In all cases the perturbation is $T^{\prime}=\cos \{6 \lambda\} \operatorname{sech}^{2}$ $\{6(\phi-\pi / 4)\}$, where $\lambda$ is longitude.

[22] Acknowledgment. This work is funded, in part, by a grant from the National Science Foundation.

\section{References}

Ambaum, M. H. P., B. J. Hoskins, and D. B. Stephenson (2001), Arctic Oscillation or North Atlantic Oscillation?, J. Clim., 14, 3495-3507.

Baldwin, M. P., and T. J. Dunkerton (1999), Propagation of the Arctic Oscillation from the stratosphere to the troposphere, J. Geophys. Res., 104, 30,937-30,946.

Baldwin, M. P., D. B. Stephenson, D. W. J. Thompson, T. J. Dunkerton, A. J. Charlton, and A. O'Neill (2003), Stratospheric memory and skill of extended-range weather forecasts, Science, 301, 636-640.

Cash, B. A., P. J. Kushner, and G. K. Vallis (2002), The structure and composition of the annular modes in an aquaplanet general circulation model, J. Atmos. Sci., 23, 3399-3414.

Charlton, A. J., A. O'Neill, W. A. Lahoz, and A. C. Massacand (2004), Sensitivity of tropospheric forecasts to stratospheric initial conditions, Q. J. R. Meteorol. Soc., in press.

Dong, B., and I. N. James (1997a), The effect of barotropic shear on baroclinic instability, part I: Normal mode problem, Dyn. Atmos. Oceans, 25, 143-167. 
Dong, B., and I. N. James (1997b), The effect of barotropic shear on baroclinic instability, part II: The initial value problem, Dyn. Atmos. Oceans, 25, 169-190.

Hartley, D. E., J. T. Villarin, R. X. Black, and C. A. Davis (1998), A new perspective on the dynamical link between the stratosphere and troposphere, Nature, 391, 471-474.

Hartmann, D. L. (2000), The key role of lower-level meridional shear in baroclinic wave life cycles, J. Atmos. Sci., 57, 389-401.

Kushner, P. J., and L. M. Polvani (2004), Stratosphere-troposphere coupling in a relatively simple AGCM: The role of eddies, J. Clim., 17, 629-639.

Perlwitz, J., and N. Harnik (2003), Observational evidence of a stratospheric influence on the troposphere by planetary wave reflection, J. Clim., 16, 3011-3026.

Plumb, R. A., and K. Semeniuk (2003), Downward migration of extratropical zonal wind anomalies, J. Geophys. Res., 108(D7), 4223, doi:10.1029/2002JD002773.

Polvani, L. M., and P. J. Kushner (2002), Tropospheric response to stratospheric perturbations in a relatively simple general circulation model, Geophys. Res. Lett., 29(7), 1114, doi:10.1029/2001GL014284.

Rivier, L., R. Loft, and L. M. Polvani (2002), An efficient spectral dynamical core for distributed memory computers, Mon. Weather Rev., 130, $1384-1396$.
Shepherd, T. G. (2002), Issues in stratosphere-troposphere coupling, J. Meteorol. Soc. Jpn., 80, 769-792.

Song, Y., and W. A. Robinson (2004), Dynamical mechanisms for stratospheric influences on the troposphere, J. Atmos. Sci., in press.

Tanaka, H. L., and H. Tokinaga (2002), Baroclinic instability in high latitudes induced by polar vortex: A connection to the Arctic Oscillation, J. Atmos. Sci., 59, 69-82.

Thorncroft, C. D., B. J. Hoskins, and M. E. McIntyre (1993), Two paradigms of baroclinic wave life-cycle behaviour, Q. J. R. Metereol. Soc., 119, $17-55$.

U.S. Standard Atmosphere (1976), U.S. Standard Atmosphere, U.S. Government Printing Office, Washington, D.C.

A. J. Charlton and M. A. H. Wittman, 500 W. 120th Street, Room 200, Columbia University, New York, NY 10027, USA. (maw2006@columbia. edu)

L. M. Polvani, Department of Applied Physics and Applied Mathematics and Department of Earth and Environmental Sciences, Columbia University, New York, NY, USA.

R. K. Scott, NorthWest Research Associates, Inc., Bellevue, WA, USA. 